\title{
Heteropoly Acid/Nitrogen Functionalized Onion-like Carbon Hybrid Catalyst for Ester Hydrolysis Reactions
}

\author{
Wei Liu, ${ }^{[a]}$ Wei Qi, ${ }^{* a]}$ Xiaoling Guo, ${ }^{[a, b]}$ and Dangsheng Su*[a]
}

Abstract: A novel heteropoly acid (HPA)/nitrogen functionalized onion-like carbon (NOLC) hybrid catalyst was synthesized through supramolecular (electrostatic and hydrogen bond) interactions between the two components. The chemical structure and acid strength of the HPA/NOLC hybrid have been fully characterized by thermogravimetric analysis, IR spectroscopy, X-ray photoelectron spectroscopy, $\mathrm{NH}_{3}$ temperature-programmed desorption and acid-base titration measurements. The proposed method for the fabrication of the HPA/NOLC hybrid catalyst is a universal strategy for dif- ferent types of HPAs to meet various requirements of acidic or redox catalysis. The hydrophobic environment of NOLC effectively prevents the deactivation of HPA in an aqueous system, and the combination of uniformly dispersed HPA clusters and the synergistic effect between NOLC and HPA significantly promotes its activity in ester hydrolysis reactions, which is higher than that of bare PWA as homogeneous catalyst. The kinetics of the hydrolysis reactions indicate that the aggregation status of the catalyst particles has great influence on the apparent activity.

\section{Introduction}

Heteropoly acid (HPA) is a kind of nanostructured transitionmetal oxide cluster with protons as counter cations, which have a stronger acidity than conventional acids such as $\gamma-\mathrm{Al}_{2} \mathrm{O}_{3}$ and $\mathrm{H}_{2} \mathrm{SO}_{4} \cdot{ }^{[1]} \mathrm{HPA}$ has shown a high catalytic activity in many acid-catalyzed reactions and related chemical industrial processes such as the synthesis of isopropyl alcohol and the polymerization of tetrahydrofuran. ${ }^{[2]}$ The significance of HPA catalyst originates from its strong acidity and controllable composition and structure. ${ }^{[3]}$ However, most HPAs lose their catalytic activity in aqueous phase because of the formation of HPA secondary aggregates and also their poor organic compatibility as inorganic clusters. ${ }^{[4]}$ In addition, the relatively high solubility of HPAs in water makes them also disadvantageous with regard to recyclability as heterogeneous catalyst.

One promising solution to prevent the deactivation of HPA in an aqueous system is the confinement and immobilization of HPAs in nanosized hydrophobic pores or channels of the support where the contacts between HPA and water molecules

[a] W. Liu, Dr. W. Qi, X. Guo, Prof. D. Su

Shenyang National Laboratory for Materials Science

Institute of Metal Research

Chinese Academy of Sciences

72 Wenhua road, Shenyang 110016 (P. R. China)

E-mail:wqi@imr.ac.cn dssu@imr.ac.cn

[b] X. Guo

Lab of Advanced Materials \& Catalytic Engineering

Dalian University of Technology

Dalian 116024 (P. R. China)

$\square$ Supporting information for this article is available on the WWW under http://dx.doi.org/10.1002/asia.201500944. are restricted. For example, the effective combination of HPA and organically modified SBA-15 or amphiphilic surfactants could limit the contact between HPAs and water molecules, and thus prominently improve their acidic catalytic activity in an aqueous system. ${ }^{[4 b, c]}$

Nanocarbon materials, such as carbon nanotubes (CNTs) or graphenes, have been considered as promising catalyst supports owing to their advantages such as high purity, high accessibility, and the possibility of tuning the specific interactions with the active phases. ${ }^{[5]}$ Several examples for the combinations of HPAs and nanocarbon materials have already been reported. $^{[6]}$ The hydrophobic graphitic structure of carbon in these hybrid catalysts could effectively prevent the aggregation of HPAs and further enrich the organic reactants on the carbon surface. ${ }^{[6]}$ The hybrid catalysts exhibit extraordinary activity in acid or redox catalytic reactions. However, there is still great potential for the further improvement of fabrication methods for HPA/nanocarbon catalysts, such as obtaining a uniform dispersion and increasing the loading amount of HPAs.

Onion-like carbon (OLC) is a kind of spherical nanocarbon particle with a few nanometers in diameter, which is composed of layers of enclosed graphitic shells. ${ }^{[7]}$ OLC exhibits a similar graphitic structure and thermal stability as multiwalled CNTs (MWCNTs), but it has a larger specific surface area, which should be beneficial to mass transfer and loading of the active components. ${ }^{[8]}$ Herein, we report a novel HPA/nitrogenfunctionalized OLC (NOLC) hybrid as a solid acid catalyst for hydrolysis reactions. The introduction of nitrogen species on the OLC surface could establish electrostatic interactions between the NOLC support and HPA clusters for an improvement of the dispersion and loading amount. The synergistic effects between the two components ensure an optimal acid catalytic 
activity and water tolerance of HPAs in ethyl acetate (EA) hydrolysis reactions. Furthermore, the proposed method for the fabrication of HPA/NOLC hybrid catalyst has been proven to be a universal strategy for both nanocarbons and HPAs, and it is applicable for other types of HPAs to meet various requirements of acidic or redox catalysis.

\section{Results and Discussion}

Scheme 1 illustrates the concept and preparation process of HPA/NOLC hybrids. The OLC samples are firstly obtained through thermal treatment with commercial ultra-dispersed diamond (UDD) at $1300^{\circ} \mathrm{C}$ in an argon atmosphere. ${ }^{[9]}$ After subsequent liquid phase oxidation ${ }^{[10]}$ and gas phase nitrogen func-

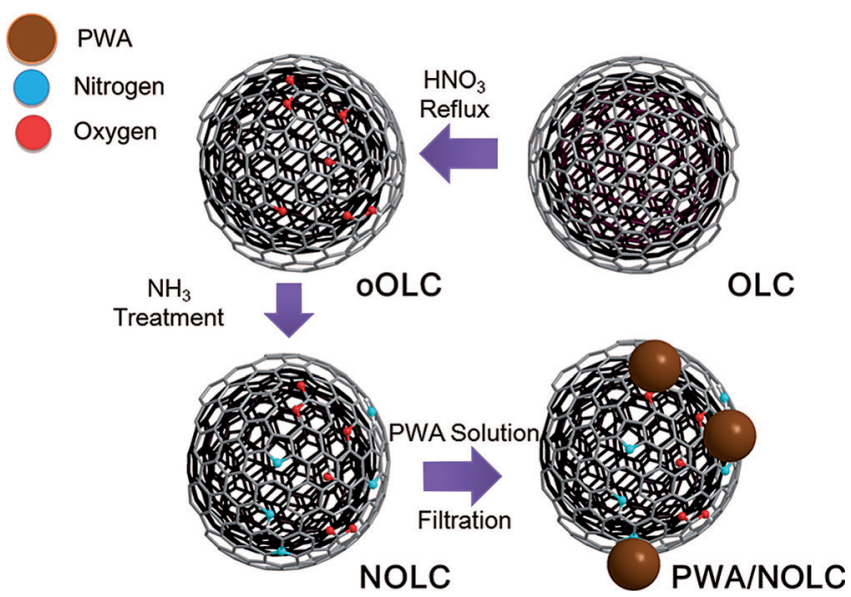

Scheme 1. Schematic illustration of the synthesis of the PWA/NOLC catalyst.

tionalization ${ }^{[11]}$ with concentrated nitric acid and ammonia, respectively, the NOLC support is eventually obtained. The vibration modes at $1577 \mathrm{~cm}^{-1}$ and $1234 \mathrm{~cm}^{-1}$ in the attenuated total reflection Fourier transform IR (ATR-FTIR) spectra of NOLC (Figure 1) are assigned as stretching vibrations of graphitic structure and carbon-nitrogen bond, respectively, confirming the success of the carbon graphitic shell formation ${ }^{[12]}$ and the nitrogen functionalization. ${ }^{[13]}$ The X-ray photoelectron spectroscopy (XPS) measurement shows that the surface atomic content of nitrogen reaches $4.5 \%$ for NOLC sample (Table S1, Sup-

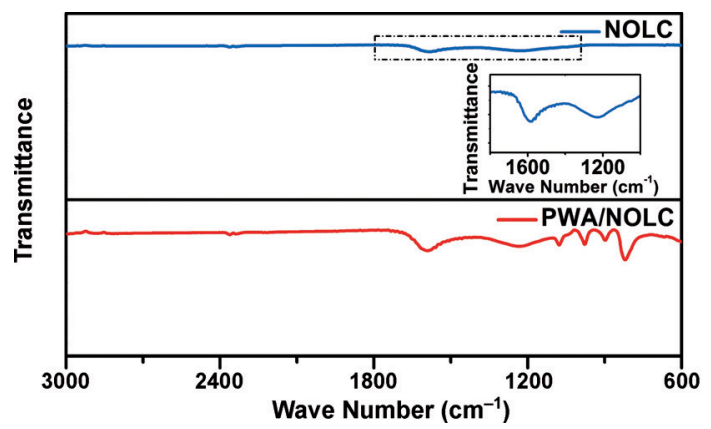

Figure 1. ATR-FTIR spectra of NOLC and PWA/NOLC. porting Information). The nitrogen atoms are incorporated into the graphitic lattice in the form of pyridinic, pyrrolic, and graphitic nitrogen species and so on, and the introduction of these species could effectively adjust the surface charge of the carbon supports. ${ }^{[11]}$

The surface charge measurements at different $\mathrm{pH}$ values suggest that the point of zero charge (PZC) of NOLC is at 5.7 (Figure S1, Supporting Information), indicating that the NOLC support is negatively charged under neutral conditions and further suggesting the success of the nitrogen functionalization. Keggin-type phosphotungstic acid (PWA) was firstly applied as a typical HPA for the hybrid catalyst fabrication. The $\mathrm{pH}$ of the PWA solution for fabrication is below 1, which means that the surface of NOLC has a positive charge under the combination conditions, which is favorable for the immobilization of the negatively charged PWA anions on the NOLC support through electrostatic interactions.

The morphology of PWA/NOLC was examined by TEM (Figure $2 \mathrm{~A}$ ). The graphitic structure of OLC can be clearly ob-

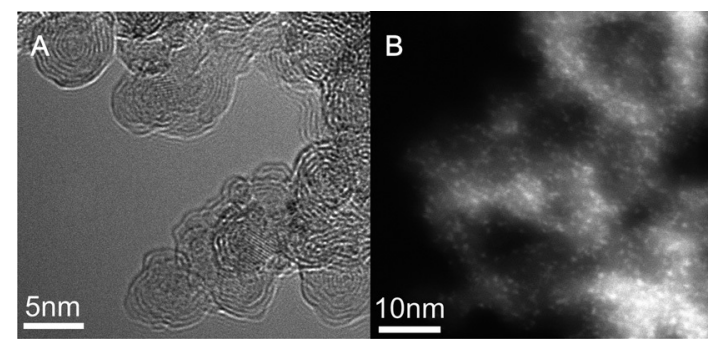

Figure 2. (A) TEM image of PWA/NOLC; (B) STEM image of PWA/NOLC.

served, and the overall morphology of PWA/NOLC is similar to that of OLC (Figure S2, Supporting Information), which means that the damage to the surface after immobilization is negligible. The fact that no PWA aggregates can be observed in the TEM images suggests a good dispersion of PWA on NOLC. In the scanning transmission electron microscopy (STEM) image, the existence of PWA clusters can be clearly confirmed as white spots (Figure 2B). The supported PWA clusters have a diameter of about $1 \mathrm{~nm}$, which is consistent with the size of a single PWA cluster $(1.2 \mathrm{~nm})$, confirming that the PWA is monodispersed on the NOLC. In addition, the lack of the PWA signal in the X-ray diffraction (XRD) pattern of PWA/NOLC also confirms the uniform dispersion of PWA clusters (Figure S3). ${ }^{[14]}$ The high dispersion of PWA clusters is beneficial for their performance in catalytic reactions.

The nature of the interactions between PWAs and NOLC supports was revealed by using XPS (Figure 3). Unlike nitrogen-doped nanocabons synthesized through chemical vapor deposition (CVD) methods, which contain little or negligible oxygen on the surface, a considerable content of oxygen functional groups is present on the surface of NOLC (oxygen content of about 4 at.\%) owing to the $\mathrm{HNO}_{3}$ treatment process. ${ }^{[15]}$ Therefore, the NOLC support may interact with PWA clusters through both oxygen and nitrogen functional groups. In the $\mathrm{N}$ 1s XPS spectrum of the PWA/NOLC hybrid, it is found that the 

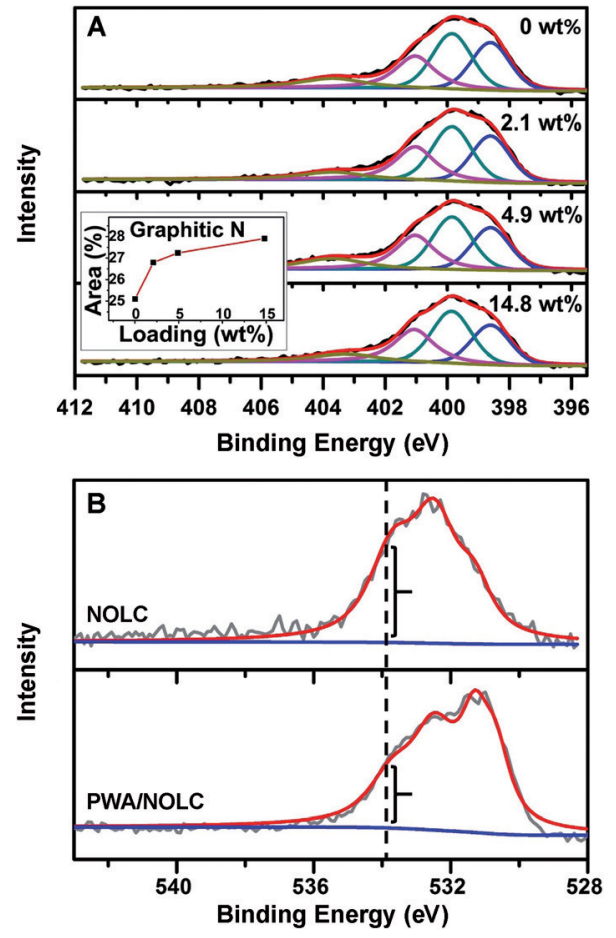

Figure 3. (A) N 1s XPS of PWA/NOLC; inset: content of graphitic N (area percentage of overall $\mathrm{N}$ content) as a function of PWA loading; (B) O 1s XPS of NOLC before and after PWA immobilization.

graphitic nitrogen content $(401.0 \mathrm{eV})$ increases along with PWA loading, which agrees with previously reported observations that HPA anions could connect with graphitic nitrogen through electrostatic interactions. ${ }^{[6]} \mathrm{A}$ decrease of the intensity at about $534 \mathrm{eV}$ in $\mathrm{O}$ 1s XPS after immobilization suggests the interactions between PWAs and oxygen functionalities, while the new peak at about $531 \mathrm{eV}$ could be assigned to the contribution of oxygen species in PWA clusters. ${ }^{[16]}$ This phenomenon is consistent with other independent studies, which suggest that HPAs would interact strongly with oxygen functional groups on carbon materials through hydrogen bonding. ${ }^{[17]}$ These two kinds of supramolecular interactions (electrostatic interactions and hydrogen bonding) existing between PWAs and oxygen/ nitrogen functionalities strengthen the connections between the two components effectively, further leading to a robust hybrid catalyst.

The ATR-FTIR spectra of PWA/NOLC (Figure 1) clearly exhibit the characteristic vibration modes of Keggin-type PWA clusters (between 800 and $1100 \mathrm{~cm}^{-1}$ ). Among them, the vibrations of $\mathrm{W}-\mathrm{O}_{c}-\mathrm{W}, \mathrm{W}-\mathrm{O}_{d}$ and $\mathrm{P}-\mathrm{O}_{\mathrm{a}}$ in PWA/NOLC are identical to those of bare PWA clusters $\left(820 \mathrm{~cm}^{-1}\right.$ vs. $818 \mathrm{~cm}^{-1}, 978 \mathrm{~cm}^{-1}$ vs. $978 \mathrm{~cm}^{-1}$, and $1078 \mathrm{~cm}^{-1}$ vs. $1076 \mathrm{~cm}^{-1}$ ), but there is a $15 \mathrm{~cm}^{-1}$ red-shift of the $\mathrm{W}^{-} \mathrm{O}_{\mathrm{b}}-\mathrm{W}$ vibration band after PWA immobilization on NOLC $\left(898 \mathrm{~cm}^{-1}\right.$ vs. $\left.913 \mathrm{~cm}^{-1}\right) \cdot{ }^{[4 c, 18]}$ This red-shift indicates the interactions of PWA with the functional groups of NOLC, and it is consistent with previous reports. ${ }^{[17]}$

Thermogravimetric analysis (TGA) under oxygenous atmosphere was applied to investigate the thermal stability of PWA/ NOLC and also to calculate the content of PWA. PWA/NOLC hy-
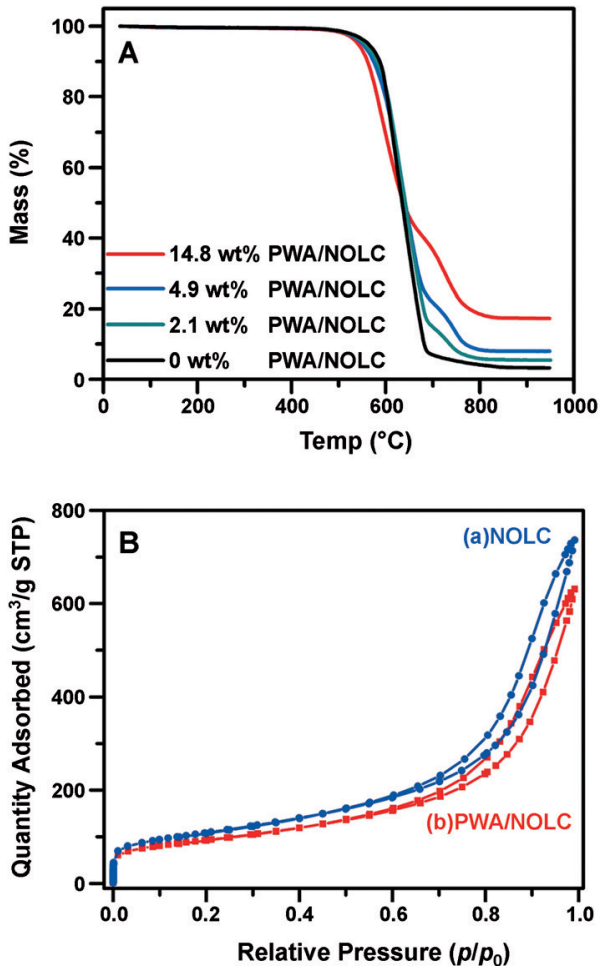

Figure 4. (A) TG curves of NOLC and PWA/NOLC with different loading; (B) $\mathrm{N}_{2}$ adsorption isotherms of (a) NOLC and (b) PWA/NOLC.

brids with different PWA loading amounts all exhibit a relatively high stability below $600^{\circ} \mathrm{C}$ (Figure 4). A dramatic weight loss occurs above $600^{\circ} \mathrm{C}$ because of the combustion of carbon. The NOLC support shows a similar stability under the same test conditions, which indicates that the PWAs have a negligible influence on the structure and stability of NOLC supports. The burned ash of PWA/NOLC is $\mathrm{WO}_{3}$, while the ash of NOLC is negligible. The loading amount of PWA in PWA/NOLC is about $15 \mathrm{wt} . \%$, which means that there are about 12 PWA clusters on average on each NOLC sphere. This loading amount is much higher than that in a previous report using CNTs as support (about 5 wt. \%). ${ }^{[6]}$ It is also the maximum PWA loading in current research. In addition, the Brunauer-Emmett-Teller (BET) surface area of the hybrid decreases by $15 \%$ after immobilization (NOLC: $377.4 \mathrm{~m}^{2} \mathrm{~g}^{-1}$ vs. PWA/NOLC: $322.5 \mathrm{~m}^{2} \mathrm{~g}^{-1}$ ) because of the high molecular weight and loading amount of PWA (Figure 4).

The superficial acid-base property of the hybrid catalysts was firstly investigated by ammonia temperature-programmed desorption $\left(\mathrm{NH}_{3} \mathrm{TPD}\right)$, as shown in Figure $5 \mathrm{~A}$. The desorption peaks centered below $400^{\circ} \mathrm{C}$ for bare PWA reflect desorbed $\mathrm{NH}_{3}$ combined with PWA via hydrogen bonding $\left(\mathrm{NH}_{2}-\mathrm{H} \cdots \mathrm{O}\right)$, while the peak centered at $570^{\circ} \mathrm{C}$ is attributed to the desorption of chemically adsorbed ammonia on PWA. For NOLC supports, the peaks below $400^{\circ} \mathrm{C}$ originate from the desorption of the weakly chemisorbed $\mathrm{NH}_{3}$. The peak centered above $500^{\circ} \mathrm{C}$ is a result of the decomposition of nitrogen species in the form of $\mathrm{NH}_{3}$, which has been also reported in a previous work. ${ }^{[6]}$ The $\mathrm{NH}_{3}$ TPD profile of PWA/NOLC exhibits the charac- 

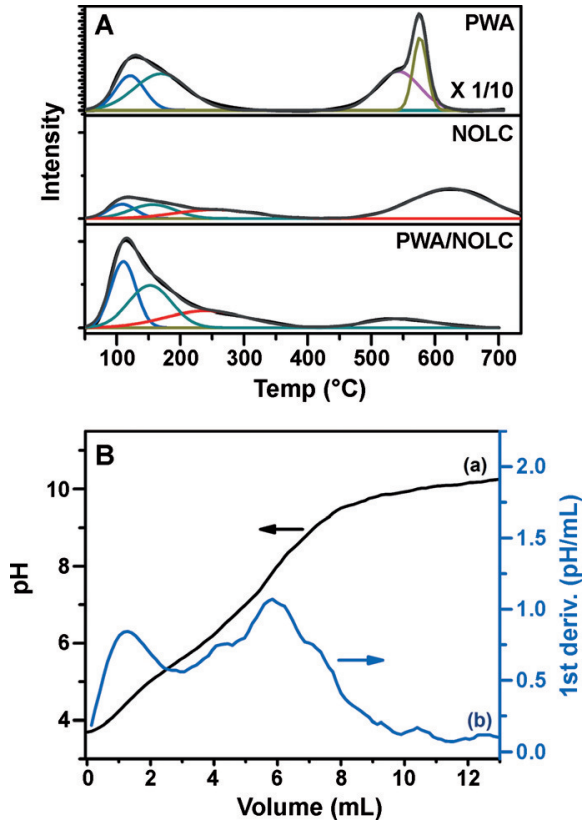

Figure 5. (A) $\mathrm{NH}_{3}$ TPD profiles of PWA, NOLC and PWA/NOLC; (B) Titration curve of PWA/NOLC. (a) $\mathrm{pH}$ vs. $\mathrm{NaOH}$ consumption, (b) The first order derivative of (a).

teristic desorption peaks of both PWA and NOLC. The integrated peak area below $400^{\circ} \mathrm{C}$ increases by $173 \%$ (NOLC: $0.60 \times$ $10^{-7}$ vs. PWA/NOLC: $1.65 \times 10^{-7}$ ), while some strong acid sites (desorption of $\mathrm{NH}_{3}$ above $500^{\circ} \mathrm{C}$ ) for PWAs are lost after immobilization. This phenomenon is also observed in the case of PWAs supported on mesoporous molecular sieves, ${ }^{[19]}$ which may be due to the interactions between PWA clusters and NOLC supports. The average number of available protons on each PWA cluster was determined to 1.9 protons per PWA through careful comparison of the $\mathrm{NH}_{3}$ desorption peak areas of PWA, NOLC and PWA/NOLC. This value is below the stoichiometric number of PWA clusters (3.0 protons per PWA), indicating that the interactions between PWAs and the functional groups hinder some protons, which means that some protons of PWA cannot be used in the acid-catalyzed reaction in an aqueous system.

Quantitative acid-base titration was also used to evaluate the acidity of the PWA/NOLC hybrid catalyst in aqueous phase. Figure $5 \mathrm{~B}$ illustrates the dependence of $\mathrm{pH}$ on the cumulative consumption of the titrant $(\mathrm{NaOH})$ and its first order derivative. The $\mathrm{pH}$ of the PWA/NOLC dispersion increases with the addition of $\mathrm{NaOH}$ titrant, and two peaks can be observed from the first order derivative profile of the $\mathrm{pH}$ trend, which means there are two kinds of acid sites in PWA/NOLC. The first peak, which is located at around $\mathrm{p} K_{\mathrm{a}}=4.5$, represents the free protons $\left(\mathrm{H}^{+}\right)$, while the second one locating at $\mathrm{p} K_{\mathrm{a}}$ around 7.8 could be assigned as protons linking with NOLC substrates; this also indicates that the acid strength of the second type of acid site is very weak. The significantly larger amount of weak acidic sites compared to strong ones (2.28X $10^{-4} \mathrm{~mol} \mathrm{H}^{+} \mathrm{g}^{-1}$ vs. $0.64 \times 10^{-4} \mathrm{~mol} \mathrm{H}^{+} \mathrm{g}^{-1}$ ) is consistent with the $\mathrm{NH}_{3}$ TPD results. Because of the similarity in the size of PWA and NOLC sphere and the uniform dispersion of PWA, it is reasonable to assume that one PWA cluster connects not only to a single NOLC sphere but multiple NOLCs.

Oxidized OLC (OOLC), which has little nitrogen but massive oxygen functional groups on its surface, was also used as support to immobilize PWA (PWA/oOLC) as a reference to evaluate the role of nitrogen functional groups. In the O 1s XPS spectra of PWA/OOLC and OOLC (Figure S4), a decrease of the intensity at about $534 \mathrm{eV}$ after immobilization is observed, indicating the interactions between PWA and the oxygen functionalities. The PWA loading of PWA/OOLC from TGA (Figure S5) is significantly lower than that of PWA/NOLC (6.0 wt.\% vs. 14.8 wt.\%), suggesting that the interactions between PWA and OOLC are weaker than those between PWA and NOLC, which further suggests the critical role of nitrogen functional groups in immobilizing PWAs.

$\mathrm{H}_{3} \mathrm{PMo}_{12} \mathrm{O}_{40}(\mathrm{PMOA})$ and $\mathrm{H}_{4} \mathrm{SiW}_{12} \mathrm{O}_{40}$ (SiWA), which have different central or addenda atoms compared with PWA, were also used to prepare hybrid catalysts (PMoA/NOLC and SiWA/ NOLC, respectively) following the synthesis strategy shown in Scheme 1. The clear signals in Mo 3d and W 4f XPS of the corresponding hybrid catalysts confirm the successful immobilization of different HPA clusters on NOLC (Figure S6). The TG and XPS characterization results (Figures S7 and S8) confirm that PMoA/NOLC and SiWA/NOLC catalysts exhibit a structure similar to PWA/NOLC hybrids, and further suggest the universality of the proposed fabrication strategy for HPA/NOLC hybrid catalysts for various HPAs.

The acid catalytic activity and water tolerance of the hybrid catalyst were evaluated by its performance in EA hydrolysis reactions (Scheme 2). $\mathrm{H}_{2} \mathrm{O}$ is not only the reactant but also the solvent for this reaction. Three types of HPA/NOLC (PWA/ NOLC, PMoA/NOLC and SiWA/NOLC) were tested, and the per-

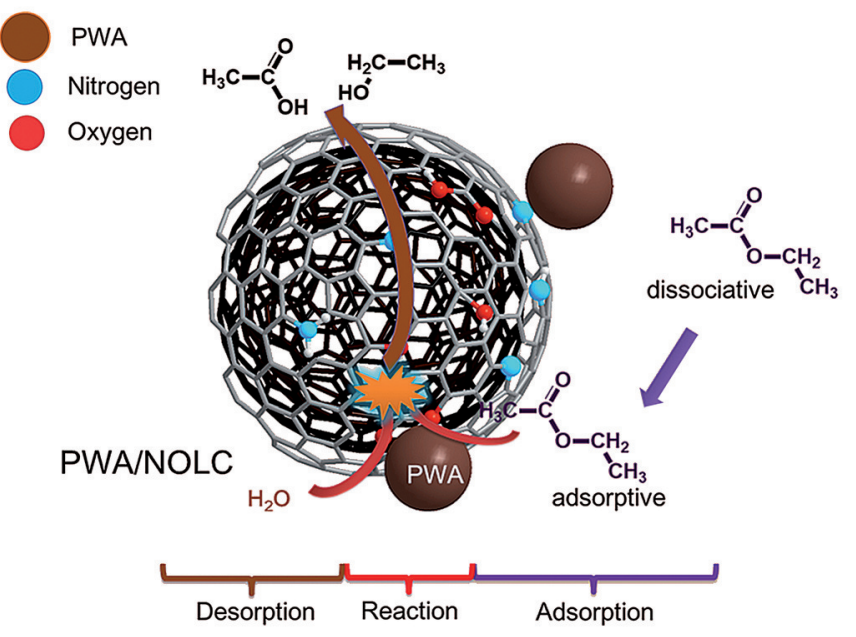

Scheme 2. Schematic illustration outlining the hydrolysis process of EA catalyzed by PWA/NOLC. 
formance of PWA/OOLC and bare PWA were also examined as references. The detected products of EA hydrolysis are only ethanol and acetic acid. The activity of various acid catalysts in EA hydrolysis reactions, including PWA/NOLC and some other classic acid catalysts, are listed in Table 1 . All the catalytic experiments were performed at $60^{\circ} \mathrm{C}$ under similar conditions. ${ }^{[4 b]}$ As shown in Table 1, the activity of PWA/oOLC (per catalyst

graphitic structure of NOLC can adsorb nonpolar EA molecules far more effectively than the hydrophilic PWAs, and the enrichment of EA surrounding the catalytic centers facilitates the catalytic hydrolysis reactions. The release of polar hydrolysis product is also easily accomplished on the graphitic surface of NOLC (Scheme 2). Similar results were also reported using modified CNTs or SBA-15 as supports. ${ }^{[46,6]}$ This close cooperation between NOLC and PWA greatly increases the catalytic efficiency of PWA.

The reusability and stability of PWA/NOLC in EA hydrolysis reactions was tested as shown in Figure S9. More than $82 \%$ of the apparent activity of PWA/ NOLC remains after the second run, and there is no further activity drop in the following reaction cycles (third and fourth reaction runs). The TGA result shows that the PWA loading amount of PWA/NOLC deceases by about $20 \%$ after the first run ( $14.8 \mathrm{wt} . \%$ vs. $11.7 \mathrm{wt} . \%$ ), but there is no further leaking of PWAs after the second run (Figure S10). In addition, the N 1s XPS spectrum of the recovered PWA/NOLC catalysts shows negligible changes (Figure S11), indicating that the interactions between PWA and nitrogen functional groups are relatively stable during reactions. As a reference, the apparent activity of PWA/

weight) is much lower than that of PWA/NOLC, which is consistent with the lower PWA loading in PWA/OOLC than PWA/ NOLC. In addition, NOLC shows a very low activity in the reaction compared with HPA/NOLC hybrid catalysts, and the activity should originate from its weak surface acidity as illustrated by $\mathrm{NH}_{3}$ TPD and acid-base titration measurements. Sulfuric acid and H-ZSM-5 zeolite, which is a traditional homogenous catalyst and a common solid acid catalyst, respectively, exhibit a relative low activity because of the deactivation of acid sites in aqueous phase. $\mathrm{SO}_{4}{ }^{2-} / \mathrm{ZrO}_{2}$ shows an acceptable activity, which may come from the unique surface chemical property of the supports that could protect the active components from $\mathrm{H}_{2} \mathrm{O}$ deactivation.

The intrinsic activity (TOF, activity per proton) of PWA/NOLC is higher than that of all other catalysts. Compared with bare PWAs, the TOF of PWA/NOLC hybrid catalyst is 1.5 times higher, even though the former one constitutes a homogeneous catalytic reaction system, indicating that the problems of PWA's secondary aggregates with $\mathrm{H}_{2} \mathrm{O}$ is well alleviated by the relatively strong interactions between PWA and NOLC. ${ }^{[4]}$ The TOF of PWA/OOLC is similar to that of PWA/NOLC, indicating that the enhanced PWA activity mainly originates from the hydrophobic nature of the support with a graphitic structure.

The reason for the high activity of the immobilized PWAs in the PWA/NOLC hybrid over unsupported ones may be the uniform dispersion of PWA and the synergistic effect between NOLC and PWAs. HPA clusters could form vesicle-like superstructure aggregates in many solvents such as water, ${ }^{[20]}$ which would decrease the surface area of HPA (the surface area of PWA is about $8 \mathrm{~m}^{2} \mathrm{~g}^{-1}$ in $\mathrm{H}_{2} \mathrm{O}$ ) and restrict their activity. In our catalyst, the PWAs are monodispersed on NOLC with a large surface area of $322.5 \mathrm{~m}^{2} \mathrm{~g}^{-1}$, which greatly increases the accessibility of PWAs to the reactants. In addition, the hydrophobic oOLC drastically drops by $41 \%$ at its second run, with the PWA loading amount significantly deceasing from $6.0 \mathrm{wt} . \%$ to $3.2 \mathrm{wt}$ \% (Figure S12), indicating that the interactions between PWA and oxygen functional groups might be vulnerable. The activity drop of the recovered PWA/NOLC in its second run may be a result of the leaking of PWA clusters that connected with oxygen functional groups during the reactions and the subsequent washing procedures; however, the activity keeps constant after the leaking of these weakly bonded PWA clusters.

The kinetics of EA hydrolysis catalyzed by HPA/NOLC are shown in Figure 6. Among all the HPA/NOLC samples, PWA/ NOLC exhibits the highest activity, while PMoA/NOLC shows the lowest activity, which is consistent with the sequence of the intrinsic acidity of the unsupported HPAs (acidity: PWA $>$ SiWA $>$ PMoA). ${ }^{[21]}$ It should be noted that aqueous phase EA hydrolysis catalyzed by HPAs is normally considered a firstorder kinetic process, ${ }^{[22]}$ while our proposed HPA/NOLC catalysts seem to behave differently as shown in Figure 6. The ap-

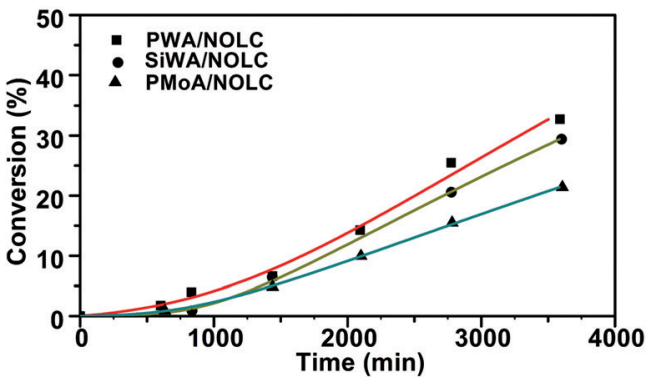

Figure 6. Kinetics of the hydrolysis of EA catalyzed by PWA/NOLC, SiWA/ NOLC and PMoA/NOLC. 
parent activity for all the hybrid catalysts increases continuously during the catalytic tests. The reasons for such an abnormal kinetic behavior might be a change of the HPA/NOLC aggregated status (namely, the number of the accessible active sites) during the catalytic process. As shown in Figure S13, the average size of PWA/NOLC clusters decreases with increasing EA conversion, since the nanosized NOLC spheres can easily aggregate to minimize their surface energy ${ }^{[23]}$ and the polarity of the reaction system significantly increases at high conversion (the hydrolysis product ethanol and acetic acid has obviously a higher polarity than the substrate EA). The change in the aggregation status of HPA/NOLC will lead to a variation of the quantity of the accessible active sites, which further influences the apparent activity and the kinetic behavior of the HPA/ NOLC hybrid.

\section{Conclusions}

In conclusion, we have prepared a novel HPA/NOLC hybrid solid acid catalyst, which has shown a promising acid catalytic activity due to the synergistic effect of the two components. The structure of HPA clusters remains intact during the immobilization process. The HPA clusters are monodispersed on the NOLC support, with their content even reaching $15 \mathrm{wt} . \%$. The HPAs interact with both oxygen and nitrogen functional groups on the surface of NOLC supports. The strong interactions between HPAs and nitrogen functional groups ensure the relatively high stability of the hybrid catalysts. The NOLC support not only enables a uniform dispersion but also provides a hydrophobic environment for HPAs to enrich organic substrates, which leads to a higher activity than that of conventional acid catalysts such as $\mathrm{H}_{2} \mathrm{SO}_{4}$ and $\mathrm{H}-\mathrm{ZSM}-5$ zeolites. To the best of our knowledge, it is the first time that NOLC has been used as support to prepare non-noble metal catalysts, and the kinetic data show that NOLC holds great potential as catalyst support.

\section{Experimental Section}

\section{Preparation of NOLC}

First, ultra-dispersed diamond (UDD) was heated at $1300^{\circ} \mathrm{C}$ in an argon atmosphere for $6 \mathrm{~h}$ to afford OLC. Then, OLC $(1 \mathrm{~g})$ was collected and refluxed in $100 \mathrm{~mL} \mathrm{HNO}_{3}(68 \%)$ at $120^{\circ} \mathrm{C}$ for $2 \mathrm{~h}$. The resulting oxidized OLC, labelled as OOLC, was filtered and washed with deionized water until the $\mathrm{pH}$ of the filtrate reached 7. Subsequently, the oOLC was dried at $120^{\circ} \mathrm{C}$ overnight. Finally, oOLC was heated in a mixture of $10 \% \mathrm{NH}_{3}$ in an argon atmosphere at $400^{\circ} \mathrm{C}$ for $4 \mathrm{~h}$ to achieve nitrogen functionalization. The resulting sample is denoted as NOLC.

\section{Preparation of HPA/NOLC}

In a typical experiment, NOLC (1 g) was first dispersed in deionized water $(250 \mathrm{~mL})$ under vigorous stirring. Then an aqueous solution of $\mathrm{H}_{3} \mathrm{PW}_{12} \mathrm{O}_{40}(150 \mathrm{~mL})$ was added dropwise to the NOLC dispersion. The mixed solution was then stirred for $6 \mathrm{~h}$. The resulting sample was washed with deionized water to remove unbounded PWA and dried overnight at $80^{\circ} \mathrm{C}$. The obtained sample is denoted as PWA/NOLC. The fabrication of SiWA/NOLC, PMoA/NOLC and PWA/NOLC at different loading content followed a similar strategy with only changing the type and feed ratio of HPA and NOLC.

\section{Catalytic experiments}

The hydrolysis of EA was carried out in a $150 \mathrm{~mL}$ thick-wall pressure vessel with mechanical stirring at $60^{\circ} \mathrm{C}$. In a typical experiment, EA (7.5 g), $\mathrm{H}_{2} \mathrm{O}(140 \mathrm{~mL}, 7.77 \mathrm{~mol})$, catalyst $(812.5 \mathrm{mg})$ and 1,4-dioxane $(500 \mu \mathrm{L}, 5.90 \mathrm{mmol})$ as internal standard were added into the vessel. A small amount of the solution $(1 \mathrm{~mL})$ was taken periodically for gas chromatography (GC) analysis. After the reaction, the catalyst was filtered off, washed with deionized water, and dried at $150^{\circ} \mathrm{C}$ for $8 \mathrm{~h}$. The conversion of EA to acetic acid and ethyl alcohol was determined by the consumption of the substrate using GC. The GC system used in this work was an Agilent 7890A system equipped with an FID detector.

\section{Characterizations}

TEM and STEM analyses were performed using an FEI Tecnai G2 F20 microscope with an accelerating voltage of $200 \mathrm{kV}$. XRD data were obtained using a D/Max-2400 X-ray diffractometer. The size distribution tests were performed by using a Malvern Nano-ZS90 ZETA sizer. TGA was performed on a NETZSCH STA449F3 instrument under a mixed flow of $\operatorname{Ar}\left(20 \mathrm{~mL} \mathrm{~min}^{-1}\right)$ and synthetic air $\left(30 \mathrm{~mL} \mathrm{~min}{ }^{-1}\right)$ at a heating rate of $10^{\circ} \mathrm{C} \mathrm{min}^{-1}$ from 35 to $950^{\circ} \mathrm{C}$. The $\mathrm{NH}_{3}$ TPD was performed on a fixed bed reactor equipped with a mass spectrometer under helium $\left(50 \mathrm{~mL} \mathrm{~min}^{-1}\right)$ at a heating rate of $5^{\circ} \mathrm{C} \mathrm{min}^{-1}$ from 35 to $750^{\circ} \mathrm{C}$. Before heating, samples $(50 \mathrm{mg}$ ) were exposed to excess $10 \% \mathrm{NH}_{3}$ in $\mathrm{Ar}\left(100 \mathrm{~mL} \mathrm{~min}^{-1}\right.$ for $\left.2 \mathrm{~h}\right)$, and the system was blown with inert gas ( $\mathrm{He}, 50 \mathrm{~mL} \mathrm{~min}^{-1}$ for $8 \mathrm{~h}$ ) at $35^{\circ} \mathrm{C}$. The XPS measurements were carried out in an ultra-high vacuum (UHV) ESCALAB 250 system equipped with a monochromatic $\mathrm{Al}_{\mathrm{K} \alpha} \mathrm{X}$-ray source $(1486.6 \mathrm{eV}$; anode operating at $15 \mathrm{kV}$ and $20 \mathrm{~mA}$ ). The specific surface area was measured by using the BET method using nitrogen adsorption-desorption isotherms on a Micrometrics ASAP 2020 system. The ATR-FTIR spectra were recorded using a Varian spectrometer equipped with a liquid nitrogencooled MCT detector. The titration of the acid strength was done with a Mettler DL77 titrator with $0.01 \mathrm{M} \mathrm{NaOH}$ as titrant.

\section{Acknowledgements}

The authors thank Dr. Bingsen Zhang for TEM and STEM support. This work was financially supported by NSFC of China (21303226, 21133010, 51221264, 21261160487), "Strategic Priority Research Program" of the Chinese Academy of Sciences and General Financial Grant from the China Postdoctoral Science Foundation (2012M520651).

Keywords: heterogeneous catalysis - heteropoly acid hydrolysis · onion-like carbon · support

[1] T. Okuhara, N. Mizuno, M. Misono, Appl. Catal. A 2001, 222, 63-77.

[2] a) C. L. Hill, Chem. Rev. 1998, 98, 1-2; b) I. V. Kozhevnikov, J. Mol. Catal. A 2007, 262, 86-92.

[3] T. Okuhara, N. Mizuno, M. Misono, Adv. Catal. 1996, 41, 113-252.

[4] a) A. Micek-Ilnicka, J. Mol. Catal. A 2009, 308, 1-14; b) K. Inumaru, T. Ishihara, Y. Kamiya, T. Okuhara, S. Yamanaka, Angew. Chem. Int. Ed. 2007, 46, 7625-7628; Angew. Chem. 2007, 119, 7769-7772; c) W. Qi, Y. Z. Wang, W. Li, L. X. Wu, Chem. Eur. J. 2010, 16, 1068-1078. 
[5] a) P. Serp, E. Castillejos, ChemCatChem 2010, 2, 41-47; b) D. S. Su, S Perathoner, G. Centi, Chem. Rev. 2013, 113, 5782-5816; c) G. Charron, A. Giusti, S. Mazerat, P. Mialane, A. Gloter, F. Miserque, B. Keita, L. Nadjo, A Filoramo, E. Riviere, W. Wernsdorfer, V. Huc, J. P. Bourgoin, T. Mallah, Nanoscale 2010, 2, 139-144.

[6] W. Qi, W. Liu, S. Y. Liu, B. S. Zhang, M. X. Gu, L. X. Guo, S. D. Su, Chem CatChem 2014, 6, 2613-2620.

[7] V. L. Kuznetsov, A. L. Chuvilin, Y. V. Butenko, I. Y. Mal'kov, V. M. Titov, Chem. Phys. Lett. 1994, 222, 343-348.

[8] N. Sano, H. Wang, I. Alexandrou, M. Chhowalla, K. B. K. Teo, G. A. J. Amaratunga, K. limura, J. Appl. Phys. 2002, 92, 2783-2788.

[9] Y. V. Butenko, V. L. Kuznetsov, A. L. Chuvilin, V. N. Kolomiichuk, S. V. Stankus, R. A. Khairulin, B. Segall, J. Appl. Phys. 2000, 88, 4380-4388.

[10] A. J. Plomp, D. S. Su, K. P. d. Jong, J. H. Bitter, J. Phys. Chem. C 2009, 113, 9865-9869.

[11] S. Kundu, W. Xia, W. Busser, M. Becker, D. A. Schmidt, M. Havenith, M. Muhler, Phys. Chem. Chem. Phys. 2010, 12, $4351-4359$.

[12] C. Branca, F. Frusteri, V. Magazu, A. Mangione, J. Phys. Chem. B 2004, $108,3469-3473$.

[13] a) T. Saito, K. Matsushige, K. Tanaka, Phys. B 2002, 323, 280-283; b) E. Mironov, A. Koretz, E. Petrov, Diamond Relat. Mater. 2002, 11, 872-876.

[14] T. Okuhara, H. Watanabe, T. Nishimura, K. Inumaru, M. Misono, Chem. Mater. 2000, 12, 2230-2238.

[15] H. B. Wang, T. Maiyalagan, X. Wang, ACS Catal. 2012, 2, 781-794.
[16] S. Ullah, J. J. S. Acuña, A. A. Pasa, S. A. Bilmes, M. E. Vela, G. Benitez, U. P. Rodrigues-Filho, Appl. Surf. Sci. 2013, 277, 111-120.

[17] a) M. A. Schwegler, P. Vinke, M. van der Eijk, H. van Bekkum, Appl. Catal. A 1992, 80, 41-57; b) Y. Kim, S. Shanmugam, ACS Appl. Mater. Interfaces 2013, 5, 12197-12204; c) J. P. Tessonnier, S. Goubert-Renaudin, S. Alia, Y. Yan, M. A. Barteau, Langmuir 2013, 29, 393-402.

[18] M. Fournier, R. Thouvenot, C. Rocchiccioli-Deltcheff, J. Chem. Soc. Faraday Trans. 1991, 87, 349-356.

[19] A. Jha, A. C. Garade, S. P. Mirajkar, C. V. Rode, Ind. Eng. Chem. Res. 2012 51, 3916-3922.

[20] H. L. Li, H. Sun, W. Qi, M. Xu, L. X. Wu, Angew. Chem. Int. Ed. 2007, 46, 1300-1303; Angew. Chem. 2007, 119, 1322-1325.

[21] B. B. Bardin, S. V. Bordawekar, M. Neurock, R. J. Davis, J. Phys. Chem. B 1998, 102, 10817-10825.

[22] C. A. Lane, M. F. Cheung, G. F. Dorsey, J. Am. Chem. Soc. 1968, 90, 64926494.

[23] A. Krüger, F. Kataoka, M. Ozawa, T. Fujino, Y. Suzuki, A. E. Aleksenskii, A. Y. Vul, E. Ōsawa, Carbon 2005, 43, $1722-1730$

Manuscript received: September 7, 2015

Final Article published: November 25, 2015 